\title{
Advanced Spectral Imaging for Microanalysis of Cultural Heritage
}

\author{
F.G France, ${ }^{*}$ M.B. Toth, ${ }^{* *}$ W.A Christens-Barry, ${ }^{* * *}$ and K. Boydston****
}

* Preservation Research and Testing Division, Library of Congress, 101 Independence Ave, SE, Washington D.C. 20540-4560

** R.B. Toth Associates, 10606 Vale Road, Oakton, VA 22124

*** Equipoise Imaging LLC, 4009 St. Johns Lane, Ellicott City, MD 21042

**** MegaVision Inc, P.O. Box 60158, Santa Barbara, CA 93160

The Preservation Research and Testing Division at the Library of Congress established an advanced spectral imaging program for preservation research and analysis of the diverse range of cultural heritage objects in its collection. Adaptations to the spectral imaging system have enabled the acquisition of detailed high resolution images of up to $6000 \mathrm{ppi}$, in effect facilitating hyperspectral microscopy (figure 1). This capacity has led to the capture and discovery of previously unknown information about important cultural and historic objects.

The Library of Congress collection numbers over 145 million items, including Thomas Jefferson's handwritten copy of the Declaration of Independence, and the L'Enfant Plan of Washington DC. Within the Preservation Research and Testing Division (PRTD), the Library has established an optical imaging laboratory that includes a spectral imaging program as a key component, including a hyperspectral imaging system. The Library is currently developing integrated research methodologies for expanding preservation analytical capabilities through non-destructive hyperspectral imaging of cultural objects, in conjunction with associated non-invasive portable analytical instrumentation [1].

Spectral imaging is undertaken with a MegaVision-Equipoise imaging system using a 39 Megapixel monochrome camera (7216x5412) E6 back, and APO-Digitar 5, 6/120 lens. The object is illuminated in specific wavebands through the visible and non-visible regions of the spectrum, included 365nm (ultra-violet), 450-638nm (visible), and 700-1050nm (infrared), as well as sidelighting with $470 \mathrm{~nm}$ (blue) and $910 \mathrm{~nm}$ (infrared). The lighting and camera system is optimized to minimize damage to documents with illumination at specific wavelengths and capture of the images with the monochrome camera, rather than illuminating the manuscript with a full spectrum light source and then filtering at the camera [2]. Integrated MegaVision Photoshoot software controls imaging and acquisition of data and associated metadata. A 16-bit per pixel, multiple image data cube is generated from the integrated set of narrowband images, acquired in sequence with the software that controls the illumination and camera, using the optimized imaging parameters.

The standard 600 ppi spectral images are now used for non-invasive materials characterization of cultural heritage items, both substrate (paper, parchment, photographic materials) and media used to record information (inks, pigments colorants. This allows the identification of key areas of interest, including areas of deterioration, previous conservation treatments, and the impact of degradative environmental parameters. Based on the 600 ppi spectral images, the key areas of interest are then imaged at 10 times the magnification, at $6000 \mathrm{ppi}$. This allows the capture of detailed images of these areas to guide preservation of the object. 
The successful integration of spectral imaging for preservation of cultural heritage objects requires ongoing collaboration across a range of scientific and cultural heritage personnel: imaging and preservation scientists, art historians, curators and conservators. Innovative imaging, processing and analysis techniques establish spectral imaging as the initial non-invasive analysis and documentation step for cultural heritage analyses. Mapping spectral responses, organic and inorganic data, and topographic microscopic imaging has greatly extended this capacity from a simple image capture technique. Generating a spectral map of all components in the object allows materials that are visibly similar to be fully characterized from spectral differences. Linking hyperspectral data with other non-invasive micro-analytical techniques further enhances the research potential of this image analysis technique. In addition, with the spectral range of illumination, visible and non-visible information can be captured in a cube of fully registered images. The development of a spectral reference collection of scientific samples is an ongoing initiative at the Library.

Further adaptations to the existing system are underway and will enhance the non-destructive utility of spectral imaging through filtering of fluorescence reflectance and emission, the inclusion of polarizing filters to further characterize cultural heritage materials, and the integration of Z-plane imaging to assess layers of media and other materials. The capacity to advance non-destructive characterization of our cultural heritage preserves the original items for future generations and allows greater access to the digital images and data for researchers and scholars.

\section{References}

[1] F.G. France et al., Proc. SPIE. 7531-13 (2010) 1-12.

[2] W.A. Christens-Barry et al., Proc. SPIE. 7249-8 (2009) 1-10.

[4] This research was supported by Deanna Marcum, Associate Librarian, Dianne van der Reyden, Director of the Preservation Directorate, and Dr. Eric Hansen, Chief PRTD. The expertise of Dr. Roger Easton, (Rochester Institute of Technology) is gratefully acknowledged.
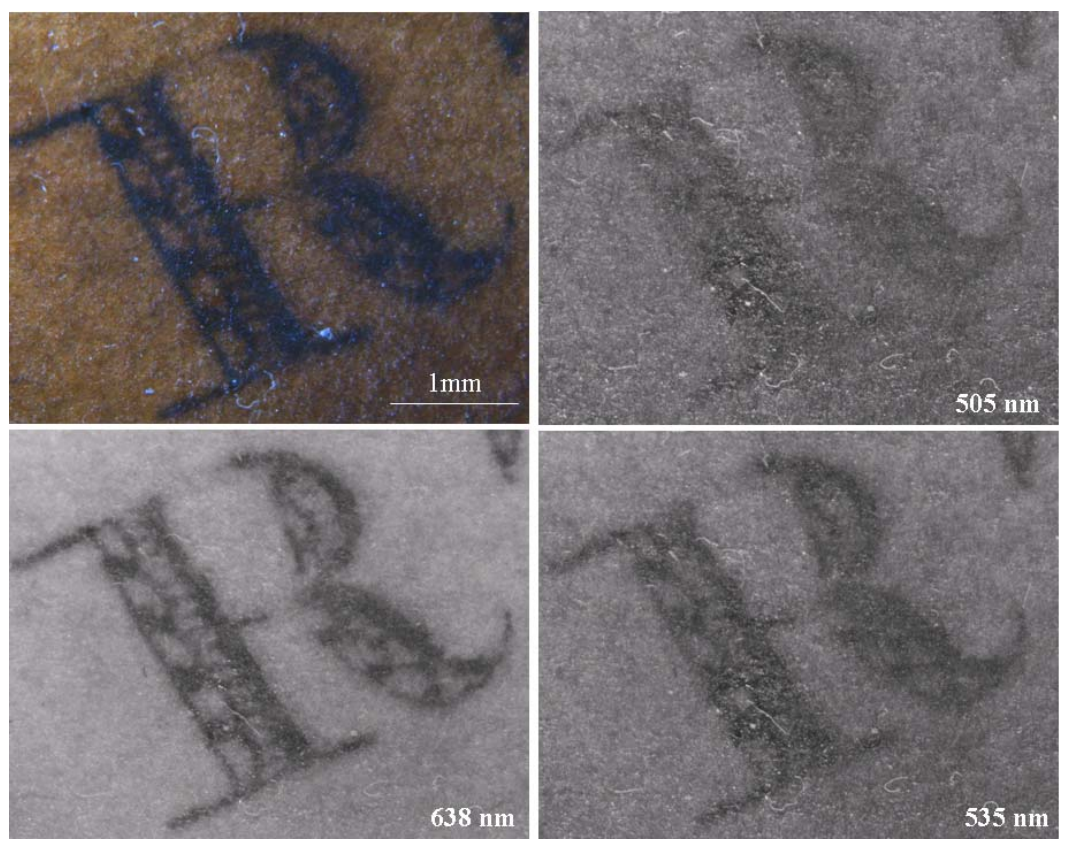

FIG. 1. Clockwise from upper left: Reflected light microscopy image of iron gall ink letter, 4000 ppi spectral images at $505 \mathrm{~nm}, 538 \mathrm{~nm}$ and $638 \mathrm{~nm}$ respectively. 